\title{
Efficient approach to nucleation and growth dynamics: Stationary diffusion flux model
}

\author{
Dennis S. van Putten ${ }^{1,2, a)}$ and Vitaly I. Kalikmanov ${ }^{1,3}$ \\ ${ }^{1}$ Twister Supersonic Gas Solutions, Einsteinlaan 10, 2289 CC, Rijswijk, The Netherlands \\ ${ }^{2}$ Department of Mechanical Engineering, University of Twente, Drienerlolaan 5, \\ 7522 NB Enschede, The Netherlands \\ ${ }^{3}$ Department of Geotechnology, Delft University of Technology, Stevinweg 1, \\ 2628 CN Delft, The Netherlands
}

(Received 19 February 2009; accepted 27 March 2009; published online 23 April 2009)

\begin{abstract}
A new model describing the evolution of clusters in the processes of nucleation and growth is proposed. The diffusion flux in the nonstationary Fokker-Planck equation with an unknown distribution function is approximated by the closed form expression containing the steady-state solution of the Zeldovich-Frenkel equation. This is justified due to the smallness of induction time of cluster formation compared to the time scale observed in experiments. The resulting stationary diffusion flux model is valid for all cluster sizes, computationally efficient and applicable to various types of cluster formation processes. Its application to a nucleation pulse experiment shows an excellent agreement with the solution of the set of formally exact Becker-Döring equations.

(C) 2009 American Institute of Physics. [DOI: 10.1063/1.3120489]
\end{abstract}

\section{INTRODUCTION}

Modeling the cluster size distribution, describing the evolution of clusters in the processes of nucleation and growth, is an important subject in various areas of physics. Applications can be found in the fields of condensing vapors, ${ }^{1}$ crystallization, ${ }^{2}$ ferromagnetics, ${ }^{3}$ aerosol and atmospheric science, ${ }^{4}$ combustion science, ${ }^{5}$ and chemical processes. ${ }^{6}$ In these fields an accurate solution of the Becker-Döring (BD) equations, or its continuous equivalent the Fokker-Planck equation (FPE), is mandatory.

The rate equations for cluster evolution were derived by Becker and Döring. ${ }^{7}$ A thorough elaboration of Penrose ${ }^{8}$ proved that given the correct initial and boundary conditions the solution of the $\mathrm{BD}$ equations exists and is unique. Zeldovich $^{9}$ showed that the BD equations reduce to the FPE in the continuous limit. Both the BD equations and the FPE couple the processes of nucleation and growth, leading to excessive computation times.

The urge for computationally more tractable models led to the introduction of the general dynamic equation (GDE). ${ }^{10}$ The GDE decouples the nucleation and growth process and models nucleation by means of the Dirac $\delta$-function. This source term introduces clusters at the critical size. At fixed external conditions, however, the newly born critical clusters are in unstable equilibrium and therefore do not grow. This results in a singular cluster size distribution. Moreover, a rapid change in external conditions resulting in an increase in the critical size leads to evaporation of all clusters. Recent studies made an attempt to overcome these deficiencies by replacing the $\delta$-function with a boundary condition at a certain size larger than the critical. ${ }^{11}$ However, the location of this boundary is not specified explicitly. Furthermore, addi-

${ }^{a)}$ Electronic mail: dennis.vanputten@twisterbv.com. tional ad hoc constraints on the size distribution in the subcritical region are introduced.

The aim of this paper is to present a simplification of the FPE, which is valid for the entire cluster range without the need for additional restraints. In general, the flux in the FPE contains a drift term and a diffusion term. The approximation refers to the diffusion flux, which is pronounced in the domain of cluster sizes up to a certain size slightly exceeding the critical cluster. It transports the newly formed clusters to the supercritical range and becomes negligibly small compared to the drift flux outside this domain. ${ }^{12}$ Due to the extreme smallness of the induction time of nucleation ${ }^{13} t_{\text {ind }}$, $\left(\sim 10^{-6} \mathrm{~s}\right)$ compared to laboratory time scales, the size distribution in the diffusion flux can be replaced by the stationary solution of Zeldovich-Frenkel equation. ${ }^{9,14}$ This leads to an analytical expression for the diffusion flux. The result is termed the stationary diffusion flux (SDF) model. The stationary cluster distribution can still change with time due to its implicit dependence on the external conditions and therefore the SDF is capable of treating time dependent problems.

\section{THEORY}

\section{A. Becker-Döring model and Fokker-Planck equation}

For completeness, we start with briefly summarizing the classical BD approach. In the BD model, the cluster evolution is considered as a sequence of elementary processes of attachment and detachment of monomers. ${ }^{7}$ The rate of change in the $n$-cluster number density $\rho_{n}$ is balanced by the net fluxes to the $n$-clusters $J_{n-1}$, and from them, $J_{n}$, as 


$$
\frac{d \rho_{n}}{d t}=J_{n-1}-J_{n}, \quad \text { for } n=2, \ldots, N,
$$

where the number density of the monomers $\rho_{1}$ is given by a boundary condition. The flux is constructed by considering the forward rate $f_{n}$ and backward rate $b_{n}$,

$$
J_{n}=f_{n} \rho_{n}-b_{n+1} \rho_{n+1} .
$$

The forward rate depends on the physics of cluster formation and the backward rate is determined from the detailed balance condition at constrained equilibrium

$$
b_{n}=f_{n-1} \frac{\rho_{n-1}^{\mathrm{eq}}}{\rho_{n}^{\mathrm{eq}}},
$$

where $b_{n}$ is assumed to be independent of the vapor density; $\rho_{n}^{\mathrm{eq}}$ is the equilibrium cluster distribution

$$
\rho_{n}^{\mathrm{eq}}=\rho_{1} e^{-g(n)}, \quad \text { with } g(n)=\frac{\Delta G(n)}{k_{B} T},
$$

where $\Delta G(n)$ is the free energy of cluster formation, $k_{B}$ is the Boltzmann constant, and $T$ is the temperature. In the continuous limit the BD model reduces to the FPE (Ref. 9) for the continuous number density $\rho(n, t)$,

$$
\frac{\partial \rho(n, t)}{\partial t}=-\frac{\partial J(n)}{\partial n},
$$

with

$$
J(n)=-f(n) \frac{\partial \rho(n, t)}{\partial n}+\dot{n}(n) \rho(n, t) .
$$

The first term on the right-hand side (rhs) of Eq. (6) is the diffusion flux in size space while the second one is the drift flux containing the growth law $\dot{n}(n) \equiv d n / d t$. The diffusion coefficient $f(n)$ and the growth law $\dot{n}(n)$ are related by the fundamental Zeldovich relation ${ }^{14}$

$$
f(n)=-\frac{\dot{n}(n)}{g^{\prime}(n)}, \quad \text { with } \quad g^{\prime}(n)=\frac{d g(n)}{d n} .
$$

It is important to note that at the critical cluster size $n^{*}$, the $g(n)$ is at maximum implying that $g^{\prime}\left(n^{*}\right)=0$. The growth rate at the critical size $\dot{n}\left(n^{*}\right)$ also vanishes; however $f\left(n^{*}\right)$ remains finite. The general form of the growth law can be written in terms of the reduced radius $r \equiv\left(n / n^{*}\right)^{1 / 3}$ as in Ref. 15,

$$
\dot{r}(r)=\frac{1}{\tau r^{\alpha}}\left(1-\frac{1}{r}\right), \quad \text { with }\left.\tau^{-1} \equiv \frac{d \dot{n}}{d n}\right|_{n^{*}},
$$

where the power index $\alpha$ depends on the type of mass exchange, e.g., $\alpha=0$ and 1 correspond to the ballistic and diffusion limited growth, respectively.

\section{B. Stationary diffusion flux model}

The starting point of the SDF is the Fokker-Planck Eqs. (5) and (6). We propose an approximation in which the diffusion term in Eqs. (5) and (6) involving the unknown cluster distribution $\rho(n, t)$ is approximated by

$$
Q_{s}=\frac{\partial}{\partial n}\left\{f(n) \frac{\partial \rho_{s}(n)}{\partial n}\right\}
$$

containing the stationary flux of nucleating clusters; here $\rho_{s}(n)$ is the nonequilibrium stationary cluster distribution. The latter is defined as the steady-state solution of the Zeldovich-Frenkel equation $^{14}$

$$
\frac{\partial}{\partial n}\left\{f(n) \rho^{\mathrm{eq}}(n) \frac{\partial}{\partial n}\left(\frac{\rho_{s}(n)}{\rho^{\mathrm{eq}}(n)}\right)\right\}=0,
$$

where $\rho^{\mathrm{eq}}(n)$ is the continuous equivalent of Eq. (4). Integrating Eq. (10) using the boundary conditions $\rho_{s}(1) / \rho^{\mathrm{eq}}(1)=1$ and $\lim _{n \rightarrow \infty}\left[\rho_{s}(n) / \rho^{\mathrm{eq}}(n)\right]=0$ yields $^{14}$

$$
\rho_{s}(n)=\rho^{\mathrm{eq}}(n) J \int_{n}^{\infty} \frac{d \widetilde{n}}{f(\tilde{n}) \rho^{\mathrm{eq}}(\tilde{n})},
$$

where the steady-state nucleation rate $J$ is

$$
J=\left[\int_{1}^{\infty} \frac{d \tilde{n}}{f(\tilde{n}) \rho^{\mathrm{eq}}(\tilde{n})}\right]^{-1} .
$$

Combining Eq. (9) with Eq. (6) written for the stationary conditions $\rho(n, t)=\rho_{s}(n)$ and $J=$ const, we find

$$
Q_{s}=\frac{\partial}{\partial n}\left\{\dot{n} \rho_{s}\right\}=\frac{\partial}{\partial n}\left\{\dot{n} \rho^{\mathrm{eq}}[1-J \varepsilon(n)]\right\},
$$

with

$$
\varepsilon(n) \equiv \int_{1}^{n} \frac{d \tilde{n}}{f(\widetilde{n}) \rho^{\mathrm{eq}}(\widetilde{n})} .
$$

This leads to the general expression for the SDF

$$
\frac{\partial \rho}{\partial t}+\frac{\partial}{\partial n}(\dot{n} \rho)=\frac{\partial}{\partial n}\left\{\dot{n} \rho^{\mathrm{eq}}[1-J \varepsilon(n)]\right\},
$$

which is valid for all cluster sizes. In the limit of "small" $n \ll n^{*}$, such that $g(n) \ll g\left(n^{*}\right)$, the free energy $g(n)$ is dominated by the positive surface contribution implying that the number of small clusters continues to have its equilibrium value in spite of the flux.

One can simplify Eq. (15) in the domain $n \geq n^{*}$ using the properties of $\rho^{\mathrm{eq}}(n)$. The diffusion coefficient is proportional to the cluster size as $n^{(2-\alpha) / 3}$ (e.g., for the ballistic growth law $\left.1 / f(n) \propto n^{-2 / 3}\right)$. Therefore, in view of the exponential function in $\rho^{\mathrm{eq}}(n)$, the main contribution of the diffusion coefficient in the integral of Eq. (11) is at the lower integration boundary, yielding

$$
\rho_{s}(n) \approx J \frac{e^{-g(n)}}{f(n)} \int_{n}^{\infty} e^{g(\widetilde{n})} d \widetilde{n} .
$$

Substituting Eq. (16) into Eq. (13) and using Eq. (7) results in

$$
Q_{s}=J \frac{\partial}{\partial n}\left\{-g^{\prime}(n) e^{-g(n)} \int_{n}^{\infty} e^{g(\widetilde{n})} d \widetilde{n}\right\} .
$$

The function $e^{g(x)}$ has a strong maximum at $x=n^{*}$, implying that we can expand $g(x)$ around $n^{*}$ up to the second order term 


$$
g(x) \approx g\left(n^{*}\right)+\frac{1}{2} g^{\prime \prime}\left(n^{*}\right)\left(x-n^{*}\right)^{2}
$$

with

$$
g^{\prime \prime}\left(n^{*}\right)=\left.\frac{d^{2} g}{d x^{2}}\right|_{n^{*}} .
$$

Substituting these relations in Eq. (17) and defining the Zeldovich factor $\mathcal{Z} \equiv \sqrt{-g^{\prime \prime}\left(n^{*}\right) / 2 \pi}$, we find

$$
Q_{s}=J \frac{\partial}{\partial n}\left\{\pi y e^{\pi y^{2}} \operatorname{erfc}[\sqrt{\pi} y]\right\}
$$

with

$$
y \equiv \mathcal{Z}\left(n-n^{*}\right),
$$

where $\operatorname{erfc}(x)$ is the complementary error function. ${ }^{16}$ For large clusters,

$$
\lim _{y \rightarrow \infty}\left\{\pi y e^{\pi y^{2}} \operatorname{erfc}[\sqrt{\pi} y]\right\}=1,
$$

yielding $Q_{s} \rightarrow 0$ for $n \rightarrow \infty$ as required. ${ }^{12}$ After simple algebra, Eq. (19) becomes

$$
Q_{s}=J \pi \mathcal{Z} \Psi(y)
$$

with

$$
\Psi(y)=-2 y+e^{\pi y^{2}}\left(1+2 \pi y^{2}\right) \operatorname{erfc}[\sqrt{\pi} y] .
$$

At the critical size $\Psi(y=0)=1$ and decreases rapidly with $y$, demonstrating the vanishing of the diffusion term at large $n$. Finally, in the supercritical domain the SDF [Eq. (15)] reduces to

$$
\frac{\partial \rho}{\partial t}+\frac{\partial}{\partial n}(\dot{n} \rho)=J \pi \mathcal{Z} \Psi(y), \quad y \geq 0,
$$

where $y$ is given by Eq. (20). Equation (22) is a convenient approximate form for nucleation experiments, in which the supercritical clusters are of major interest. The rhs represents the source term with newly born clusters and shows resemblance with the GDE, ${ }^{10}$

$$
\frac{\partial \rho}{\partial t}+\frac{\partial}{\partial n}(\dot{n} \rho)=J \delta\left(n-n^{*}\right) .
$$

The difference, however, is that the source term of Eq. (22) takes into account all supercritical clusters and not just $n^{*}$. This prevents the appearance of a singular size distribution for fixed external conditions.

The general SDF [Eq. (15)] is applicable to all $1 \leq n$ $<\infty$ and is solved by imposing an initial condition and by specifying the thermodynamic model of cluster formation $g(n)$ and the growth law $\dot{n}(n)$. Moreover, no additional constraints are necessary for the subcritical clusters.

\section{RESULTS AND DISCUSSION}

We apply the SDF to a nucleation pulse experiment (see, e.g., Refs. 17 and 18). The test case consists of a typical stepwise constant pressure-temperature profile comprising a region with high supersaturation, where nucleation and growth take place (region I), followed by a region with low
TABLE I. Nucleation pulse test case conditions for water with nonequilibrium vapor molar fraction $y_{w}=7 \times 10^{-3}$.

\begin{tabular}{lcc}
\hline \hline Parameter & Region $I(t \leq 50 \mu \mathrm{s})$ & Region II $(50 \mu \mathrm{s}<t<300 \mu \mathrm{s})$ \\
\hline$p(\mathrm{kPa})$ & 77.0 & 115.5 \\
$T(\mathrm{~K})$ & 240.0 & 270.0 \\
$S$ & 14.34 & 1.67 \\
$n^{*}$ & 23 & 1885 \\
$J\left(\mathrm{~m}^{-3} \mathrm{~s}^{-1}\right)$ & $3.96 \times 10^{16}$ & $\sim 10^{-180}$ \\
$t_{\text {ind }}(\mu \mathrm{s})$ & 1.7 & 29.2 \\
\hline \hline
\end{tabular}

supersaturation where nucleation is negligible (region II). An example of such an experiment is presented in Table I. The pulse duration is $50 \mu \mathrm{s}$. The supersaturation is given by $S$ $=y_{w} p / p_{\text {sat }}(T)$, where $p$ is the total pressure, $y_{w}$ is the nonequilibrium vapor molar fraction, and $p_{\text {sat }}(T)$ is the saturation pressure of the nucleating substance. We take water as a nucleating substance with $y_{w}=7 \times 10^{-3}$; its thermodynamic properties are those of Ref. 17. The induction time of nucleation is defined as $t_{\text {ind }}=\left(4 \pi f\left(n^{*}\right) \mathcal{Z}^{2}\right)^{-1}$ (see, e.g., Ref. 13).

The results are validated against the solution of the set of formally exact $\mathrm{BD}$ [Eq. (1)], where the forward rate is determined from gas kinetics. ${ }^{14}$ The backward rate is obtained from Eq. (3) using the Frenkel free energy of formation ${ }^{14}$

$$
g(n)=-n \ln S+\theta_{\infty} n^{2 / 3}
$$

with

$$
\theta_{\infty}=\frac{\gamma_{\infty} s_{1}}{k_{B} T}, \quad s_{1}=(36 \pi)^{1 / 3}\left(\rho_{l}\right)^{-2 / 3},
$$

where $\gamma_{\infty}$ is the plain layer surface tension and $\rho_{l}$ is the liquid number density. The monomer density is nondepleting and assumed constant in both regions. A set of $10^{5} \mathrm{BD}$ equations is solved using the piecewise constant flux approximation. ${ }^{19}$ The SDF is solved by the van Leer MUSCL scheme (see Ref. 20 and references therein). Using the SDF model, the computational effort is reduced by a factor of 8 compared to the solution of the BD equations. For consistent comparison with the BD equations, the growth law in the SDF should be chosen appropriately, ${ }^{21}$

$$
\dot{n}(n)=f(n)\left[1-\exp \left(g^{\prime}(n)\right)\right] .
$$

Figure 1 depicts the cluster size distribution in region I at several time instants. The SDF shows a good agreement with the $\mathrm{BD}$ equations for all cluster sizes. A small difference between the solutions at $t_{1}$ is due to the instantaneous occurrence of the pulse at $t=0$. The $\mathrm{BD}$ solution exhibits a certain relaxation time to be noticed in the supercritical domain. For times larger than $t_{1}$ the difference becomes negligible. At large $n$ and $t$ the diffusion term vanishes and $\rho(n, t) \approx \rho_{s}(n)$ $\approx J / \dot{n}(n)$ until the double exponential decay of the front of the size distribution occurs, as described by Shneidman. ${ }^{15}$

The results for the SDF in region II (Fig. 2) are in excellent agreement with the $\mathrm{BD}$ solutions in the entire cluster domain. The size distribution in the region of $n \ll n^{*}$ resembles the equilibrium size distribution, as observed in Eq. (15). In region II, the SDF reduces to the conservation equation for $\rho(n, t)$ in the supercritical region. We find that for the 


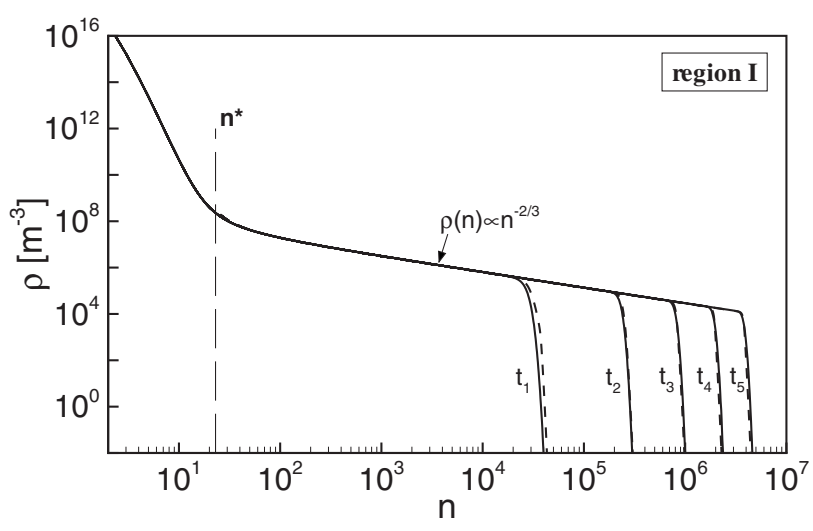

FIG. 1. Cluster size distribution $\rho(n, t)$ : BD equations (solid) vs SDF (shortdashed line) for the nucleation pulse experiment in region I at $t_{1}=10 \mu \mathrm{s}$, $t_{2}=20 \mu \mathrm{s}, t_{3}=30 \mu \mathrm{s}, t_{4}=40 \mu \mathrm{s}$, and $t_{5}=50 \mu \mathrm{s}$. The vertical long-dashed line shows the location of the critical cluster.

critical clusters $\rho\left(n^{*}, t\right)$ decays exponentially with time in agreement with Ref. 22. Furthermore, the SDF solution exhibits a general feature: the exponential decay of $\rho(n, t)$ with time is true for the subcritical clusters up to the equilibrium size distribution. This test case demonstrates the validity of the SDF for the entire cluster range.

The SDF can be used for validation of various types of nucleation experiments (e.g., nucleation pulse tubes, diffusion cloud chambers, and expanding nozzle flows ${ }^{18,23}$ ). For continuously varying pressure-temperature profiles ${ }^{23}$ all $p, T$-dependent functions- $J, S, g(n)$, and $\rho_{s}(n)$ - become parametric functions of time.

From the size distribution, the evolution of the total number of formed clusters with time can be calculated. For the nucleation pulse experiment of Table I, a portion of the clusters formed in region I evaporates $(\sim 10 \%)$. This is due to the rapid increase in the critical size in region II, causing a part of the size distribution to become subcritical. This correction can be applied to experimentally measured nucleation rates. Moreover, the SDF can serve as a guideline for designing nucleation pulse experiments. Important parameters in these experiments are the pulse duration time (region

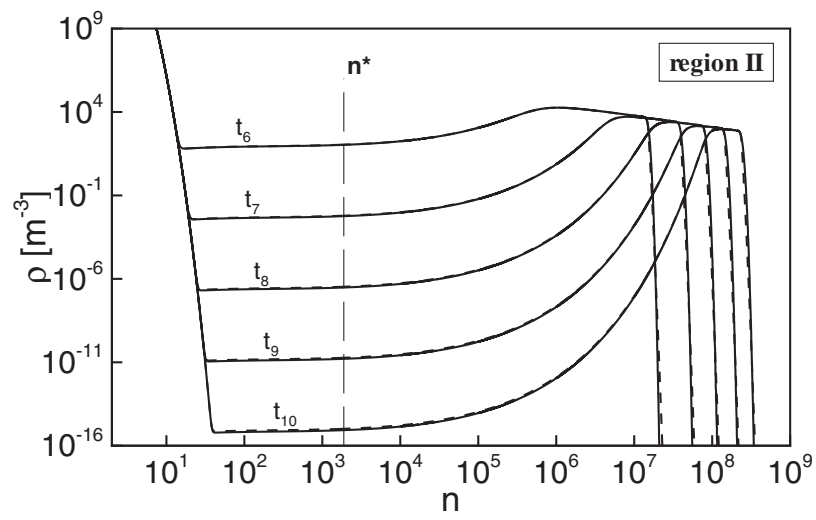

FIG. 2. Cluster size distribution $\rho(n, t)$ in region II at $t_{6}=100 \mu \mathrm{s}, t_{7}$ $=150 \mu \mathrm{s}, t_{8}=200 \mu \mathrm{s}, t_{9}=250 \mu \mathrm{s}$, and $t_{10}=300 \mu \mathrm{s}$. Other notations, see Fig. 1.
I) and ratio between the supersaturation in both regions. Additionally, an accurate description of the shape of the cluster distribution provides useful information for the applied measurement techniques.

In conclusion, the evolution of clusters in the processes of nucleation and growth can be well approximated by the SDF. The model is concise and capable of treating any time dependent problem under the assumption that the time scales of the experiment are much longer than the induction time of nucleation. The SDF does not invoke additional constraints on the cluster size distribution and can be applied to all cluster sizes. For most practical applications, in which the supercritical clusters are of major importance, the simplified SDF for $n \geq n^{*}$ can be used [Eq. (22)]. Note that the elaboration in this paper has been carried out for a purely phenomenological model of the free energy of cluster formation. Future work will focus on the extension of the SDF for alternative models of $g(n)$, e.g., mean-field kinetic nucleation theory. ${ }^{24}$ The validation of the SDF is demonstrated for a nucleation pulse experiment, but it is expected to be of general validity for any cluster formation process.

We thank R. Hagmeijer and R. Sidin for discussions and useful comments.

${ }^{1}$ R. Hagmeijer, R. H. A. IJzermans, and F. Put, Phys. Fluids 17, 056101 (2005).

${ }^{2}$ V. A. Shneidman and M. C. Weinberg, J. Non-Cryst. Solids 194, 145 (1996); L. Gránásy and P. F. James, J. Chem. Phys. 113, 9810 (2000).

${ }^{3}$ V. A. Shneidman, Physica A 190, 145 (1992).

${ }^{4}$ F. Gelbard and J. H. Seinfeld, J. Colloid Interface Sci. 68, 363 (1979).

${ }^{5}$ S. E. Pratsinis, Prog. Energy Combust. Sci. 24, 197 (1998).

${ }^{6}$ D.-M. Kim and P. D. Iedema, Chem. Eng. Sci. 63, 2035 (2008).

${ }^{7}$ R. Becker and W. Döring, Ann. Phys. 24, 719 (1935).

${ }^{8}$ O. Penrose, J. Stat. Phys. 89, 305 (1997) (and references therein).

${ }^{9}$ Ya. B. Zeldovich, Acta Physicochim. URSS 18, 1 (1943).

${ }^{10}$ J. H. Seinfeld, Atmospheric Chemistry and Physics of Air Pollution (Wiley, New York, 1986); M. M. R. Williams and S. K. Loyalka, Atmospheric Science Theory and Practice (Pergamon, New York, 1991).

${ }^{11}$ V. Holten and M. E. H. van Dongen, J. Chem. Phys. 130, 014102 (2009); R. S. R. Sidin, R. Hagmeijer, and U. Sachs, Phys. Fluids (unpublished).

${ }^{12}$ V. A. Shneidman and M. C. Weinberg, J. Non-Cryst. Solids 160, 89 (1993).

${ }^{13}$ F. F. Abraham, J. Chem. Phys. 51, 1632 (1969); D. Kashchiev, Nucleation: Basic Theory with Applications (Butterworth, Washington, DC, 2000).

${ }^{14}$ E. M. Lifshitz and L. P. Pitaevskii, Physical Kinetics (Butterworth, Washington, DC, 2006); J. Frenkel, Kinetic Theory of Liquids (Dover, New York, 1955).

${ }^{15}$ V. A. Shneidman, J. Chem. Phys. 115, 8141 (2001).

${ }^{16}$ M. Abramowitz and I. A. Stegun, Handbook of Mathematical Functions (Dover, New York, 1972).

${ }^{17}$ J. Wölk and R. Strey, J. Phys. Chem. B 105, 11683 (2001).

${ }^{18}$ C. C. M. Luijten, K. J. Bosschaart, and M. E. H. van Dongen, J. Chem. Phys. 106, 8116 (1997); R. Strey, P. E. Wagner, and Y. Viisanen, J. Phys. Chem. 98, 7748 (1994).

${ }^{19}$ D. B. Duncan and A. R. Soheili, Appl. Numer. Math. 37, 1 (2001).

${ }^{20}$ B. van Leer, J. Comput. Phys. 32, 101 (1979).

${ }^{21}$ V. A. Shneidman and W. C. Weinberg, J. Chem. Phys. 97, 3621 (1992); 97, 3629 (1992).

${ }^{22}$ V. A. Shneidman, Phys. Rev. Lett. 101, 205702 (2008).

${ }^{23}$ D. Brus, V. Ždímal, and J. Smolík, J. Chem. Phys. 129, 174501 (2008); A. Khan, C. H. Heath, U. M. Dieregsweiler, B. E. Wyslouzil, and R. Strey, ibid. 119, 3138 (2003).

${ }^{24}$ V. I. Kalikmanov, J. Chem. Phys. 124, 124505 (2006). 